Bundesgesundheitsbl 2017 $\cdot 60: 774-782$

DOI 10.1007/s00103-017-2557-2

Online publiziert: 16. Mai 2017

(c) Der/die Autor(en) 2017. Dieser Artikel ist eine Open-Access-Publikation.

CrossMark

Severine Koch · Mark Lohmann · Astrid Epp · Gaby-Fleur Böl

Bundesinstitut für Risikobewertung, Berlin, Deutschland

\title{
Risikowahrnehmung von Kontaminanten in Lebensmitteln
}

bedrohlich empfunden, da sie aus Sicht der Bevölkerung weniger der eigenen Kontrolle unterliegen und grundsätzlich als ein aufgezwungenes Gesundheitsrisiko angesehen werden [12].

Viele Fragen im Hinblick auf die Wahrnehmung von Gesundheitsrisiken durch Kontaminanten sind aus unterschiedlichen Gründen jedoch noch ungeklärt. So gibt es derzeit nur eine vergleichsweise geringe Anzahl wissenschaftlicher Studien zur öffentlichen Risikowahrnehmung gegenüber Kontaminanten in Lebensmitteln, die zudem teils geringe Stichprobengrößen aufweisen, aufgrund qualitativer Forschungsansätze [12] oder einer thematischen Zuspitzung auf einzelne Kontaminanten [13-15]. Hinzu kommt ein in zweifacher Hinsicht bedeutsames Definitionsproblem: nämlich einerseits die Verwendung unterschiedlicher Definitionen von Kontaminanten im Rahmen wissenschaftlicher Studien (so gelten in einigen Studien z. B. Rückstände von Pflanzenschutzmitteln und Antibiotika als Kontaminanten [16]) und andererseits Definitionsbarrieren zwischen Laien und Experten [17]. Bei Befragungsstudien und der partizipativen Risikokommunikation stellt die sprachliche Aufbereitung von Themen, die durch Fachterminologie geprägt sind, eine besondere Herausforderung dar. Durch die Verwendung unverständlicher und uneinheitlicher Terminologie werden Laien von vornherein von einer inhaltlichen Diskussion ausgeschlossen [10].

Die Thematiken „Ernährung und Lebensmittel“ zeichnen sich außerdem durch eine Vielzahl gesundheitsrelevanter Aspekte aus, die sich nicht auf den Bereich Kontaminanten beziehen [3]. Lebensmittelzusatzstoffe [12, 18, 19], der
Zucker- und Salzgehalt von Lebensmitteln [20], Pestizidrückstände [3] sowie die kalorische Beschaffenheit von Speisen sind möglicherweise im Bewusstsein der Bevölkerung stärker verankert als eine Belastung von Lebensmitteln mit Kontaminanten. Wesentliche Ziele der vorliegenden Repräsentativbefragung waren es, herauszufinden, inwieweit die Bevölkerung bei lebensmittelbedingten Gesundheitsrisiken spontan an Kontaminanten denkt und ob maßgebliche Vorsichtsmaßnahmen wie die Faustregel „vergolden statt verkohlen“ zur Reduzierung des Acrylamidgehalts beim Erhitzen von stärkehaltigen Lebensmitteln weiten Teilen der Bevölkerung bekannt sind. Zudem sollen die Ergebnisse einen Überblick darüber verschaffen, welche Kontaminanten der Bevölkerung geläufig sind, welchen Lebensmitteln eine hohe Belastung mit Kontaminanten nachgesagt wird und welche individuellen Maßnahmen zur Risikominimierung ergriffen werden. Allgemein bestand die Zielsetzung der Befragung darin, aktuelle Daten zur Risikowahrnehmung gegenüber Lebensmittelkontaminanten zu erheben und mögliche Anknüpfungspunkte und Handlungsbedarf für eine zielgruppengerechte Risikokommunikation $\mathrm{zu}$ identifizieren.

\section{Methoden}

\section{Stichprobe}

Hierfür wurde eine repräsentative Bevölkerungsstichprobe von 1001 Personen mittels computergestützter telefonischer Interviews (CATI-Methode) befragt [21]. Die Probanden wurden zufällig aus der Grundgesamtheit der in Privathaushalten lebenden, deutschsprachigen Bevöl- 
kerung ab 14 Jahren ausgewählt ${ }^{1}$. Die Auswahl der Befragungsperson in Haushalten mit mehreren Personen erfolgte ebenfalls per Zufallsverfahren. Dabei wurde die Person, die zuletzt Geburtstag hatte, als das zu befragende Haushaltsmitglied festgelegt (sog. Last-BirthdayMethode [22]). Da ein wachsender Anteil der Bevölkerung nur noch mobil erreichbar ist [23], wurden in der Stichprobenziehung neben Festnetznummern auch Mobilfunknummern mit einem Anteil von $20 \%$ berücksichtigt. Die Interviews hatten eine durchschnittliche Dauer von 18 min und wurden zwischen dem 17. und 26. November 2016 durchgeführt.

\section{Verwendete Terminologie und Begriffserläuterung}

Da die unter Experten gängige Terminologie für Kontaminanten in Lebensmitteln aus Sicht der Autoren als nicht laientauglich und folglich als ungeeignet für eine Bevölkerungsbefragung angesehen wird, wurde dieser Ausdruck in den Interviews durchgängig durch den Ausdruck „unerwünschte Stoffe in Lebensmitteln" ersetzt. Um eine inhaltliche Übereinstimmung der Begriffe zu gewährleisten, verlas der Interviewer im Anschluss an die Einstiegsfragen (s. Abschn. "Einleitung Fragebogen“) die folgende Begriffserläuterung: „Unter unerwünschten Stoffen verstehen wir in dieser Befragung entsprechend der wissenschaftlichen Definition Stoffe, die durch Umwelteinflüsse oder Verarbeitungsprozesse bei der Herstellung und Zubereitung unbeabsichtigt in Lebensmittel gelangen. $\mathrm{Zu}$ diesen unerwünschten Stoffen zählen beispielsweise Schwermetalle wie Blei, Cadmium oder Quecksilber bzw. Stoffe wie Dioxin oder Acrylamid. Nicht gemeint sind Stoffe, die Lebensmitteln mit Absicht zugefügt werden, wie z. B. Konservierungsstoffe oder Rückstände von Stoffen, die absichtlich bei der Produktion von Lebensmitteln eingesetzt werden, wie Pflanzenschutzmittelwirkstoffe."

\footnotetext{
1 Nach Standards des Arbeitskreises Deutscher Markt- und Sozialforschungsinstitute e. V. (ADM).
}

\section{Einleitung Fragebogen (vor Begriffserläuterung)}

Die einleitende Frage richtete sich an die generelle Kenntnis von unerwünschten Stoffen in Lebensmitteln („Haben Sie schon einmal davon gehört, dass Lebensmittel unerwünschte Stoffe enthalten können oder haben Sie davon noch nicht gehört?"). Probanden, die angaben, bereits von unerwünschten Stoffen gehört zu haben, wurden zudem gebeten, konkrete Beispiele zu benennen („Können Sie mir ein oder mehrere Beispiele für unerwünschte Stoffe in Lebensmitteln nennen?"). Diese beiden Fragen wurden der oben beschriebenen Begriffserläuterung mit der Absicht vorangestellt, spontane Nennungen von Substanzen oder Substanzgruppen zu erfassen, die die Probanden als Kontaminanten ansehen, ungeachtet der Tatsache, ob es sich dabei um Kontaminanten im wissenschaftlich-rechtlichen Sinne [1] handelt. Diese Art der Fragestellung ermöglichte es, den Anteil der Probanden zu bestimmen, der beim Thema unerwünschte Stoffe in Lebensmitteln spontan und ohne Hilfestellung seitens des Interviewers an Kontaminanten gemäß der genannten Definition dachte. Ergebnisse vorangegangener Studien deuten darauf hin, dass andere Themenfelder im Bereich Ernährung und Lebensmittel, wie beispielsweise Lebensmittelzusatzstoffe, stärker im Bewusstsein der Bevölkerung verankert sein könnten [12, 18]. Dies scheint im Wesentlichen der stärkeren gesellschaftlichen und medialen Auseinandersetzung mit diesen Themen geschuldet zu sein.

Auf die Einstiegsfragen folgte die Begriffserläuterung mit dem Ziel, einen gemeinsamen Diskussionskontext für die weitere Befragung zu schaffen, auf den sich alle nachfolgenden Fragen bezogen. Durch diese explizite Abgrenzung des Begriffs „unerwünschte Stoffe in Lebensmitteln" verengte sich der Interpretationsrahmen auf die Definition von Kontaminanten im naturwissenschaftlich-rechtlichen Sinne [2].
Fragebogen Hauptteil (nach Begriffserläuterung)

Im Hauptteil der Befragung wurden die Probanden zu einer Reihe von Themen im Zusammenhang mit unerwünschten Stoffen in Lebensmitteln befragt. • Tab. 1 zeigt eine Übersicht der verwendeten Fragen mit den jeweiligen Antwortkategorien. Die Fragen richteten sich auf die allgemeine Risikoeinschätzung der Befragten und die erwartete Entwicklung der Belastung von Lebensmitteln mit unerwünschten Stoffen (Fragen 1.1 und 1.2), den Informationsstand und die generelle Einstellung zu unerwünschten Stoffen (Fragen 2.1 und 2.2), die Vermeidung und Bewertung einzelner Lebensmittel (Hühnereier, Pistazien, Kräutertee, Honig, Reis und Reisprodukte, Milch, Äpfel, Kartoffelchips, Röstkaffee, Thunfisch, Grillfleisch) und Kontaminanten (Dioxin in Eiern oder Milch, Arsen in Reis und Reisprodukten, Acrylamid in Pommes frites oder getoastetem Brot, Benzpyren in Grillfleisch, Quecksilber in Fisch, Pyrrolizidinalkaloide in Tee und Honig; s. Frage 3.1-3.6), den Informationsbedarf der Befragten und die hauptverantwortlichen Akteure im Hinblick auf die Vermeidung gesundheitlicher Risiken durch unerwünschte Stoffe (Fragen 4.1 und 4.2).

\section{Demografische und individualisie- rende Informationen}

Um Gruppenunterschiede anhand von demografischen Charakteristiken $\mathrm{zu}$ berücksichtigen, wurden zudem Informationen zu Alter, Geschlecht, Bildung, Haushaltsgröße, Migrationshintergrund (d.h. Proband selbst oder mindestens ein Elternteil außerhalb Deutschlands geboren), Berufsgruppe und Erwerbstätigkeit der Bevölkerungsstichprobe erhoben. Darüber hinaus wurden die Probanden $\mathrm{zu}$ ihrem Einkaufsverhalten („Kaufen Sie regelmäßig Bio-Lebensmittel ein?"), ihrer Ernährungsweise („Ernähren Sie sich vegetarisch oder vegan?") und ihrem allgemeinen Risikoverhalten („Sind Sie im Allgemeinen ein risikobereiter Mensch oder versuchen Sie, Risiken zu vermeiden?") befragt. 


\section{Ergebnisse}

\section{Spontane Nennungen vor der Begriffserläuterung}

Die Daten wurden mit der Statistiksoftware SPSS (Version 21) ausgewertet. Für die kategorischen Variablen wurden statistische Unterschiede anhand von $\chi^{2}$-Tests ermittelt, als Signifikanzniveau diente dabei ein $p$-Wert von 0,05 (vgl.

- Tab. 2, 3 und 4).

Rund $92 \%$ der 1001 Befragten geben an, bereits von unerwünschten Stoffen in Lebensmitteln gehört zu haben. Erwartungsgemäß zeigt die offene Nachfrage, dass die Bevölkerung beim Thema unerwünschte Stoffe in Lebensmitteln spontan und vor der Begriffserläuterung durch den Interviewer eher selten an Kontaminanten im wissenschaftlichrechtlichen Sinne [2] denkt. Lediglich $9 \%$ der Befragten nennen mindestens einen Stoff, der laut Definition eine Kontaminante ist. Soziodemografische Unterteilungen hinsichtlich der Häufigkeit, mit der Kontaminanten spontan als Beispiele angeführt werden, sind in $\bullet$ Tab. 2 dargestellt. Die häufigsten Nennungen konform der Definition von Kontaminanten entfallen auf die Kategorien Metalle/Schwermetalle und Mineralöl (jeweils 2,3\% der Befragten, die angeben, bereits von unerwünschten Stoffen in Lebensmitteln gehört zu haben) sowie Mikroplastik (1,9\%) und Weichmacher (1,6\%).

Über die Hälfte der Befragten beziehen sich hingegen auf Stoffe, die nicht als Kontaminanten gelten. Gut $30 \%$ der Befragten führen Lebensmittelzusatzstoffe als Beispiele für unerwünschte Stoffe in Lebensmitteln an, allen voran Geschmacksverstärker (12,4\%) sowie Konservierungs- und Farbstoffe (9\% und $8,8 \%)$. Zucker $(6,8 \%)$ und Rückstände von Pestiziden (8,0\%) sowie Arzneimittel wie Antibiotika (5,5\%) werden ebenfalls häufig als unerwünschte Stoffe genannt.

\section{Risikoeinschätzung und Entwicklungstendenz (nach Begriffserläuterung)}

Knapp $60 \%$ der Befragten sehen in unerwünschten Stoffen gemäß der wis-

Bundesgesundheitsbl 2017 -60:774-782 DOI 10.1007/s00103-017-2557-2

(c) Der/die Autor(en) 2017. Dieser Artikel ist eine Open-Access-Publikation.

S. Koch · M. Lohmann · A. Epp · G.-F. Böl

\section{Risikowahrnehmung von Kontaminanten in Lebensmitteln}

\section{Zusammenfassung}

Hintergrund. Kontaminanten in Lebensmitteln können ein ernst zu nehmendes gesundheitliches Risiko darstellen. Für eine angemessene Risikokommunikation muss auch die subjektive Risikowahrnehmung der Öffentlichkeit Berücksichtigung finden. Das weite Themenspektrum und die häufig nicht konsequent vollzogene begriffliche Trennung zu Rückständen und Zusatzstoffen erfordern dabei eine umfangreiche Verdeutlichung gegenüber Verbrauchern. In einer repräsentativen Bevölkerungsbefragung wurde eine laiengerechte Sprache mit einer klaren Begriffsabgrenzung kombiniert, um die Risikoeinschätzung der Bevölkerung zu erfassen.

Ziel der Arbeit. Ziel war es, die Bekanntheit von Kontaminanten und deren wahrgenommene Gesundheitsrisiken zu bestimmen. Ermittelt wurden zudem der Informationsstand und -bedarf der Bevölkerung, die Grundeinstellungen in Bezug auf Kontaminanten, die Ansichten zu verantwortlichen Akteuren sowie die Einhaltung konkreter Schutzmaßnahmen, wie etwa die Vermeidung bestimmter
Lebensmittel, um Gesundheitsrisiken durch Kontaminanten zu reduzieren.

Methoden. Eine repräsentative Bevölkerungsstichprobe von 1001 Personen wurde mittels computergestützter telefonischer Interviews zum Thema „Kontaminanten in Lebensmitteln" befragt.

Ergebnisse. Die Mehrheit der Befragten stuft Kontaminanten als ein hohes Gesundheitsrisiko ein, obwohl nur eine Minderheit spontan Kontaminanten im Sinne der wissenschaftlich-rechtlichen Definition als Beispiele für unerwünschte Stoffe in Lebensmitteln aufführt. Quecksilber und Dioxin sind die bekanntesten Kontaminanten. Pyrrolizidinalkaloide (PA) sind der Bevölkerung nahezu unbekannt. Diskussion. Die vorliegenden Ergebnisse zeigen einen themenspezifischen Handlungsbedarf auf und bieten Anknüpfungspunkte für eine zielgruppengerechte Risikokommunikation.

Schlüsselwörter

Kontaminanten - Lebensmittel - Risiko . Wahrnehmung B Bevölkerung

\section{Perceived risks of food contaminants}

\section{Abstract}

Background. Food contaminants can pose a serious health threat. In order to carry out adequate risk communication measures, the subjective risk perception of the public must be taken into account. In this context, the breadth of the topic and insufficient terminological delimitations from residues and food additives make an elaborate explanation of the topic to consumers indispensable. A representative population survey used language adequate for lay people and a clear definition of contaminants to measure risk perceptions with regard to food contaminants among the general public. Objectives. The study aimed to assess public awareness of contaminants and the perceived health risks associated with them. In addition, people's current knowledge and need for additional information, their attitudes towards contaminants, views on stakeholder accountability, as well as compliance with precautionary measures, such as avoiding certain foods to reduce health risks originating from contaminants, were assessed.

Materials and methods. A representative sample of 1001 respondents was surveyed about food contaminants via computerassisted telephone interviewing. Results. The majority of respondents rated contaminants as a serious health threat, though few of them spontaneously mentioned examples of undesirable substances in foods that fit the scientific or legal definition of contaminants. Mercury and dioxin were the most well-known contaminants. Only a minority of respondents was familiar with pyrrolizidine alkaloids.

Conclusions. The present findings highlight areas that require additional attention and provide implications for risk communication geared to specific target groups.

Keywords

Contaminants · Food · Risk · Perception · General public 
1. Risikoeinschätzung und Entwicklungstendenz

Frage 1.1 Wenn unerwünschte Stoffe in Lebensmitteln enthalten sind: Inwiefern stellt das Ihrer Ansicht nach ein gesundheitliches Risiko dar?

Frage 1.2 Glauben Sie, dass die Belastung von Lebensmitteln mit unerwünschten Stoffen eher abnimmt, eher zunimmt oder eher gleich bleibt?

\section{Informiertheit und Einstellung}

Frage 2.1 Wie schätzen Sie Ihren eigenen Informationsstand in Bezug auf unerwünschte Stoffe in Lebensmitteln und den damit verbundenen Gesundheitsrisiken ein?

Frage 2.2 Sagen Sie mir bitte, welche der folgenden drei Aussagen am ehesten auf Sie persönlich zutrifft: (1) Ich meide bewusst Lebensmittel, denen eine hohe Belastung mit unerwünschten Stoffen nachgesagt wird.

(2) Mir ist bewusst, dass manche Lebensmittel unerwünschte Stoffe enthalten können, aber das ist nicht ausschlaggebend dafür, welche Lebensmittel ich kaufe und wie ich sie zubereite.

(3) In meinem Alltag mache ich mir keine Gedanken über unerwünschte Stoffe in Lebensmitteln

\section{Vermeidung und Bewertung einzelner Lebensmittel und Kontaminanten}

Frage 3.1 Können Sie mir auch sagen, welche Lebensmittel Sie in diesem Zusammenhang vor allem meiden $?^{\mathrm{b}}$

Frage 3.2 Inwiefern besteht Ihres Wissens bei den folgenden Lebensmitteln ein Gesundheitsrisiko durch unerwünschte Stoffe? ${ }^{\text {a } H u ̈ h n e r e i e r ; ~ P i s t a z i e n ; ~ K r a ̈ u t e r t e e ; ~ H o n i g ; ~ R e i s ~ u n d ~ R e i s p r o d u k t e ; ~ M i l c h ; ~}$ Äpfel; Kartoffelchips; Röstkaffee; Thunfisch; Grillfleisch

Frage 3.3 Ich lese Ihnen im Folgenden einige konkrete Beispiele für unerwünschte Stoffe in Lebensmitteln vor. Bitte sagen Sie mir jeweils, ob Sie schon einmal von dem Thema gehört haben oder ob Sie noch nicht davon gehört haben ${ }^{\text {a }}$ Dioxin in Eiern oder Milch; Arsen in Reis und Reisprodukten; Acrylamid in Pommes frites oder getoastetem Brot; Benzpyren in Grillfleisch; Quecksilber in Fisch; Pyrrolizidinalkaloide (kurz: PA) in Tee und Honig

Frage 3.4 Inwieweit stellt [das jeweilige Thema] Ihrer Ansicht nach ein gesundheitliches Risiko dar? Dioxin in Eiern oder Milch; Arsen in Reis und Reisprodukten; Acrylamid in Pommes frites oder getoastetem Brot; Benzpyren in Grillfleisch; Quecksilber in Fisch; Pyrrolizidinalkaloide (kurz: PA) in Tee und Honig

Frage 3.5 Ist [das jeweilige Thema] aus Ihrer Sicht etwas, dem informierte Verbraucher durch ihr eigenes Verhalten leicht entgegenwirken können oder ist es das nicht? ${ }^{c}$ Dioxin in Eiern oder Milch; Arsen in Reis und Reisprodukten; Acrylamid in Pommes frites oder getoastetem Brot; Benzpyren in Grillfleisch; Quecksilber in Fisch; Pyrrolizidinalkaloide (kurz: PA) in Tee und Honig

Frage 3.6 Was ist Ihrer Ansicht nach die wichtigste Maßnahme, um [dem jeweiligen Thema] entgegenzuwirken $^{\mathrm{d}}$ Dioxin in Eiern oder Milch; Arsen in Reis und Reisprodukten; Acrylamid in Pommes frites oder getoastetem Brot; Benzpyren in Grillfleisch; Quecksilber in Fisch; Pyrrolizidinalkaloide (kurz: PA) in Tee und Honig

4. Verantwortung und Informationsbedarf

Frage 4.1 Ich nenne Ihnen nun eine Reihe von Gruppen bzw. Organisationen. Bitte benennen Sie die wichtigste, die Ihrer Ansicht nach dafür verantwortlich ist, einer gesundheitlichen Gefährdung durch unerwünschte Stoffe in Lebensmitteln entgegenzuwirken ${ }^{\text {. }}$.

Verbraucher und Verbraucherverbände;

Wissenschaft; NGOs und Umweltverbände; Staat und öffentliche Institutionen; EU; Lebensmittelindustrie und -handel

Frage 4.2 Wie wichtig ist es Ihnen als Verbraucher, mehr über die folgenden Aspekte von unerwünschten Stoffen in Lebensmitteln zu erfahren? ${ }^{\mathrm{a}}$

(1) Informationen darüber, wie Verbraucher sich selbst vor gesundheitlichen Risiken im Zusammenhang mit unerwünschten Stoffen in Lebensmitteln schützen können, z. B. Informationen zu Zubereitungsweisen oder Einkauftipps

(2) Informationen darüber, wie unerwünschte Stoffe in Lebensmitteln rechtlich reguliert wer-

den, z. B. welche Maßnahmen der Staat dagegen ergreift

(3) Informationen zu allen Produkten, die unerwünschte Stoffe enthalten können
Skala von 1 (gar kein Risiko) bis 5 (sehr hohes Risiko)

$1=$ nimmt eher $a b$,

2 = bleibt eher gleich,

$3=$ nimmt eher $z u$

Skala von 1 (sehr schlecht) bis 5 (sehr gut)

(1) = „aktive Vermeidung“

(2) = "informierte Nichtbeachtung"

(3) $={ }_{\text {"generelle Themenferne }} "$

Kategorisierung der offenen Nennungen

Skala von 1 (gar kein Risiko) bis 5 (sehr hohes Risiko)

$1=\mathrm{Ja}$, jeweiliges Thema bekannt $2=$ Nein, jeweiliges Thema nicht bekannt

Skala von 1 (gar kein Risiko) bis 5 (sehr hohes Risiko)

$1=\mathrm{Ja}$, dem lässt sich leicht entgegenwirken

2 = Nein, dem lässt sich nicht leicht entgegenwirken

Kategorisierung der offenen Nennungen

Nennung des wichtigsten Akteurs

Skala von 1 (überhaupt nicht wichtig) bis 5 (sehr wichtig) 
Tab. 2 Spontane Nennung von Kontaminanten als unerwünschte Stoffe in Lebensmitteln: „Können Sie mir ein oder mehrere Beispiele für unerwünschte Stoffe in Lebensmitteln nennen?"

\begin{tabular}{|c|c|c|c|c|c|c|c|}
\hline & & \multicolumn{2}{|c|}{ Kontaminanten } & \multicolumn{2}{|c|}{$\begin{array}{l}\text { Andere Nennun- } \\
\text { gen }\end{array}$} & \multicolumn{2}{|c|}{$\begin{array}{l}\text { Keine Nennun- } \\
\text { gen }\end{array}$} \\
\hline & & $n$ & $\%$ & $n$ & $\%$ & $n$ & $\%$ \\
\hline \multicolumn{2}{|c|}{ Total $\left(\right.$ Basis $\left.n=925^{\mathrm{a}}\right)$} & 83 & 9 & 507 & 55 & 335 & 36 \\
\hline \multirow[t]{2}{*}{ Geschlecht } & $\mathrm{m}$ & 43 & 10 & 237 & 53 & 170 & 38 \\
\hline & w & 40 & 8 & 270 & 57 & 165 & 35 \\
\hline \multirow[t]{2}{*}{ Haushaltsgröße } & $\leq 2^{*}$ & 40 & 7 & 317 & 56 & 205 & 37 \\
\hline & $>2^{*}$ & 43 & 12 & 190 & 52 & 130 & 36 \\
\hline \multirow{2}{*}{$\begin{array}{l}\text { Migrationshin- } \\
\text { tergrund }\end{array}$} & Ja & 11 & 7 & 95 & 61 & 51 & 33 \\
\hline & Nein & 72 & 9 & 412 & 54 & 284 & 37 \\
\hline \multirow[t]{2}{*}{ Bundesländer } & Alte & 70 & 9 & 422 & 56 & 260 & 35 \\
\hline & Neue & 13 & 8 & 85 & 49 & 75 & 43 \\
\hline \multirow{2}{*}{$\begin{array}{l}\text { Einkaufsver- } \\
\text { halten }\end{array}$} & Biologisch & 45 & 11 & 245 & 61 & 110 & 28 \\
\hline & Konventionell & 38 & 7 & 259 & 50 & 225 & 43 \\
\hline \multicolumn{8}{|c|}{$\begin{array}{l}\text { alle Befragten, die bereits von unerwünschten Stoffen in Lebensmitteln gehört haben } \\
\text { *Untergruppen unterscheiden sich signifikant voneinander }\left(x^{2} \text {-Test, } p<0,05\right) \\
\text { Abweichungen von } 100 \% \text { ergeben sich durch Rundungen }\end{array}$} \\
\hline
\end{tabular}

senschaftlich-rechtlichen Definition ein (sehr) hohes gesundheitliches Risiko; etwa ein Drittel schätzt das gesundheitliche Risiko als mäßig ein. Eine Minderheit von $7 \%$ der Befragten bewertet die gesundheitlichen Risiken von unerwünschten Stoffen in Lebensmitteln als gering oder vernachlässigbar.

Die generelle Entwicklung der Belastung von Lebensmitteln mit unerwünschten Stoffen schätzt die Mehrheit der Befragten als eher zunehmend ein (58\%). Knapp ein Drittel gibt an, dass die Belastung eher gleich bleibt, während $9 \%$ der Auffassung sind, dass sie eher abnimmt. Im Vergleich zum Stichprobendurchschnitt schätzt ein statistisch signifikant größerer Anteil der Personen mit Migrationshintergrund $(n=188)$ die Belastung mit unerwünschten Stoffen als zunehmend ein (70\%). Arbeiter bewerten die Belastung überdurchschnittlich häufig als eher abnehmend ( $27 \%$, statistisch signifikant).

\section{Informiertheit und Einstellung}

Knapp die Hälfte (48\%) der Befragten gibt an, sich mittelmäßig über unerwünschte Stoffe in Lebensmitteln informiert zu fühlen, während je etwa ein Viertel der Befragten sich entweder (sehr) gut oder (sehr) schlecht informiert fühlen.
Jüngere Personen fühlen sich im Allgemeinen statistisch signifikant schlechter informiert als ältere: Bei den 14- bis 29-Jährigen gaben rund $41 \%$ an, (sehr) schlecht über unerwünschte Stoffe in Lebensmitteln informiert zu sein, im Vergleich zu $15 \%$ bei den über 60 -Jährigen.

Die Bestimmung der Grundhaltung gegenüber unerwünschten Stoffen anhand der Einteilung in "aktive Vermeidung“, „,informierte Nichtbeachtung" und "generelle Themenferne" (vgl. - Tab. 1) zeigt, dass knapp die Hälfte der Befragten (49\%) bewusst Lebensmittel meidet, denen eine hohe Belastung mit unerwünschten Stoffen nachgesagt wird. Knapp ein Drittel der Befragten (31\%) gibt an, über die Problematik informiert zu sein, aber sich dadurch nicht in ihrem Einkaufs- und Zubereitungsverhalten beeinflussen zu lassen. Etwas weniger als ein Fünftel der Befragten $(18 \%)$ machen sich in ihrem Alltag keine Gedanken über unerwünschte Stoffe in Lebensmitteln.

Insbesondere Ältere, Frauen, Personen, die Risiken generell eher vermeiden, Personen aus den alten Bundesländern und solche, die sich vegetarisch oder vegan ernähren und regelmäßig BioLebensmittel einkaufen, zeichnen sich durch eine signifikant stärkere Vermeidung belasteter Lebensmittel oder geringere Themenferne aus (s. - Tab. 3).
Die Einstellungstendenz der Befragten spiegelt sich ebenfalls in statistisch signifikanten Unterschieden bei der subjektiven Informiertheit wider. Während "Themenferne“ und „informierte Nichtbeachtung" sich in niedrigen Anteilen von Personen mit einem guten oder sehr guten Wissensstand niederschlägt (14,5\% und $16,3 \%)$, schätzen rund $34 \%$ der „aktiven Vermeider" ihren Wissensstand als gut oder sehr gut ein.

\section{Vermeidung und Bewertung einzelner Lebensmittel und Kontaminanten}

Personen, deren Grundhaltung in ,aktiver Vermeidung" besteht, führen in der offenen Nachfrage überwiegend Fleisch und Fleischprodukte (27\%) - einschließlich Wurst und Geflügel - als Beispiele für Lebensmittel an, die sie wegen ihrer (mutmaßlichen) Belastung mit unerwünschten Stoffen meiden. • Abb. 1 zeigt eine grafische Übersicht der häufigsten Nennungen. Diese Untergruppe meidet laut eigenen Angaben zudem häufig Fertigprodukte (15\%) und Lebensmittel, die aus anderen Ländern importiert wurden bzw. nicht aus regionalem Anbau stammen $(11 \%)$.

Von den 11 Lebensmittelkategorien, die hinsichtlich ihres Gesundheitsrisikos durch unerwünschte Stoffe bewertet werden, schätzen die Befragten Kartoffelchips ( $45 \%$ hohes oder sehr hohes Risiko), Grillfleisch (44\% hohes oder sehr hohes Risiko) und Thunfisch ( $40 \%$ hohes oder sehr hohes Risiko) am riskantesten ein (s. • Abb. 2). Hinsichtlich der Einschätzung des gesundheitlichen Risikos von unerwünschten Stoffen in Grillfleisch unterscheiden Frauen und Männer sich signifikant. Die Hälfte der Frauen sieht in Grillfleisch ein hohes oder sehr hohes Risiko durch unerwünschte Stoffe im Vergleich zu $37 \%$ der Männer. Fast ein Viertel der befragten Männer (23\%) sieht in Grillfleisch nur ein geringes oder gar kein Risiko, von den weiblichen Befragten teilen lediglich $14 \%$ diese Einschätzung.

Quecksilber in Fisch sowie Dioxin in Eiern oder Milch sind mit 78 und $70 \%$ die bekanntesten Kontaminanten in Lebensmitteln. Pyrrolizidinalkaloide (PA) 
Tab. 3 Allgemeine Einstellung zu unerwünschten Stoffen in Lebensmitteln (in \%): „Sagen Sie mir bitte, welche der folgenden drei Aussagen am ehesten auf Sie persönlich zutrifft". (1) Ich meide bewusst Lebensmittel, denen eine hohe Belastung mit unerwünschten Stoffen nachgesagt wird („,aktive Vermeidung"), (2) mir ist bewusst, dass manche Lebensmittel unerwünschte Stoffe enthalten können, aber das ist nicht ausschlaggebend dafür, welche Lebensmittel ich kaufe und wie ich sie zubereite („,informierte Nichtbeachtung"), (3) in meinem Alltag mache ich mirkeine Gedanken über unerwünschte Stoffe in Lebensmitteln ("generelle Themenferne")

\begin{tabular}{|c|c|c|c|c|c|c|c|c|c|}
\hline & & \multicolumn{2}{|c|}{ Aktive Vermeidung } & \multicolumn{2}{|c|}{$\begin{array}{l}\text { Informierte Nichtbe- } \\
\text { achtung }\end{array}$} & \multicolumn{2}{|c|}{ Generelle Themenferne } & \multicolumn{2}{|c|}{$\begin{array}{l}\text { Mittelwert und Stan- } \\
\text { dardabweichung }\end{array}$} \\
\hline & & $\%$ & $n$ & $\%$ & $n$ & $\%$ & $n$ & $\mathrm{M}$ & SD \\
\hline \multicolumn{2}{|c|}{ Total (Basis $n=1001)$} & 49 & 495 & 31 & 314 & 18 & 179 & 1,7 & 0,8 \\
\hline \multirow[t]{2}{*}{ Geschlecht } & $\mathrm{m}$ & 46 & 225 & 33 & 161 & 21 & 101 & 1,8 & 0,8 \\
\hline & w & 53 & 270 & 30 & 153 & 15 & 78 & 1,6 & 0,7 \\
\hline \multirow[t]{5}{*}{ Alter } & 14-29 Jahre & 22 & 46 & 38 & 79 & 39 & 81 & 2,2 & 0,8 \\
\hline & 30-39 Jahre & 39 & 54 & 43 & 59 & 15 & 21 & 1,8 & 0,7 \\
\hline & 40-49 Jahre & 64 & 112 & 22 & 39 & 13 & 22 & 1,5 & 0,7 \\
\hline & 50-59 Jahre & 60 & 106 & 27 & 48 & 12 & 20 & 1,5 & 0,7 \\
\hline & $\geq 60$ Jahre & 58 & 177 & 29 & 88 & 11 & 35 & 1,5 & 0,7 \\
\hline \multirow[t]{2}{*}{ Bundesländer } & Alte & 50 & 414 & 32 & 268 & 16 & 132 & 1,7 & 0,7 \\
\hline & Neue & 46 & 81 & 26 & 46 & 27 & 47 & 1,8 & 0,8 \\
\hline \multirow[t]{3}{*}{ Risikotyp } & Risikobereit & 34 & 88 & 38 & 98 & 27 & 69 & 1,9 & 0,8 \\
\hline & Mittel & 49 & 241 & 33 & 161 & 17 & 81 & 1,7 & 0,7 \\
\hline & $\begin{array}{l}\text { Nicht risikobe- } \\
\text { reit }\end{array}$ & 65 & 166 & 22 & 55 & 11 & 28 & 1,5 & 0,7 \\
\hline \multirow[t]{2}{*}{ Einkaufsverhalten } & Biologisch & 67 & 291 & 25 & 110 & 8 & 34 & 1,4 & 0,6 \\
\hline & Konventionell & 36 & 202 & 36 & 204 & 26 & 145 & 1,9 & 0,8 \\
\hline \multirow[t]{2}{*}{ Ernährungsform } & $\begin{array}{l}\text { Vegetarisch/ } \\
\text { Vegan }\end{array}$ & 55 & 50 & 39 & 35 & 3 & 3 & 1,5 & 0,6 \\
\hline & Konventionell & 49 & 442 & 31 & 279 & 19 & 176 & 1,7 & 0,8 \\
\hline
\end{tabular}

in Tee oder Honig (13\%) und Benzpyren in Grillfleisch (18\%) sind hingegen nur einer Minderheit der Befragten bekannt. Die Bekanntheitswerte der 6 Kontaminanten sind zusammen mit dem Gesundheitsrisiko, das in der Wahrnehmung der Befragten von ihnen ausgeht und der Kontrollierbarkeit des Risikos in - Tab. 4 dargestellt. Die Tabelle zeigt, dass Quecksilber in Fisch nicht nur das bekannteste, sondern auch das Thema mit dem höchsten vermuteten $\mathrm{Ge}$ sundheitsrisiko ist $(71 \%$ hohes oder sehr hohes Risiko). Ein systematischer, statistisch signifikanter Zusammenhang zwischen dem Bekanntheitsgrad von Kontaminanten und der Schwere des vermuteten Gesundheitsrisikos wird nicht deutlich. Die beiden Kontaminanten, die sich nach Ansicht der Befragten am ehesten durch das eigene Verhalten kontrollieren lassen, sind Benzpyren in Grillfleisch (72\%) und Acrylamid in Pommes frites oder getoastetem Brot (69\%). Die offe- ne Nachfrage zeigt, dass die Befragten bei möglichen Kontrollmaßnahmen primär an Verzicht oder eine Reduktion des Konsums denken. Im Fall von Benzpyren und Acrylamid ist aber auch die Art der Zubereitung (z. B. „Fett nicht in die Glut tropfen lassen“ oder „Toastbrot nicht zu dunkel werden lassen“) von mindestens einem Drittel der Befragten als mögliche Kontrollmaßnahme aufgeführt.

\section{Verantwortung und Informations- bedarf}

Rund ein Viertel der Befragten sieht die Lebensmittelindustrie und den Lebensmittelhandel in der Hauptverantwortung, einem gesundheitlichen Risiko durch unerwünschte Stoffe in Lebensmitteln entgegenzuwirken. Ein Fünftel benennt Verbraucherverbände als den hauptverantwortlichen Akteur, gefolgt vom Staat und anderen öffentlichen Institutionen mit $14 \%$.
Durchweg hoch ist der Informationsbedarf der Befragten im Hinblick auf (1) mögliche Schutzmaßnahmen vor unerwünschten Stoffen in Lebensmitteln, (2) die rechtliche Regulation von unerwünschten Stoffen und (3) betroffene Produkte und Produktgruppen. Zwischen 72 und $76 \%$ der Befragten geben an, es (sehr) wichtig zu finden, mehr über diese drei Aspekte von unerwünschten Stoffen in Lebensmitteln zu erfahren.

Signifikante Korrelationen zwischen dem Wissensstand der Befragten und der Wichtigkeit zusätzlicher Informationen zu den drei Aspekten von unerwünschten Stoffen zeigen, dass Personen mit höherem Wissensstand dem Erwerb weiterer Informationen eine größere Bedeutung beimessen als Personen mit niedrigerem Wissensstand ( $r=0,11$ für Informationen zu Schutzmaßnahmen, $r=0,15$ für Informationen zur rechtlichen Regulierung, $r=0,08$ für Informationen zu betroffenen Produkten; $p<0,05)$. 
Tab. 4 Bewertung konkreter Kontaminanten in Lebensmitteln hinsichtlich Bekanntheit („Bitte sagen Sie mir jeweils, ob Sie schon einmal von dem Thema gehört haben oder ob Sie noch nicht davon gehört haben“), Gesundheitsrisiko („Inwieweit stellt [das jeweilige Thema] Ihrer Ansicht nach ein gesundheitliches Risiko dar?") und Kontrollierbarkeit („Ist [das jeweilige Thema] aus Ihrer Sicht etwas, dem informierte Verbraucher durch ihr eigenes Verhalten leicht entgegenwirken können oder ist es das nicht?") in Prozent

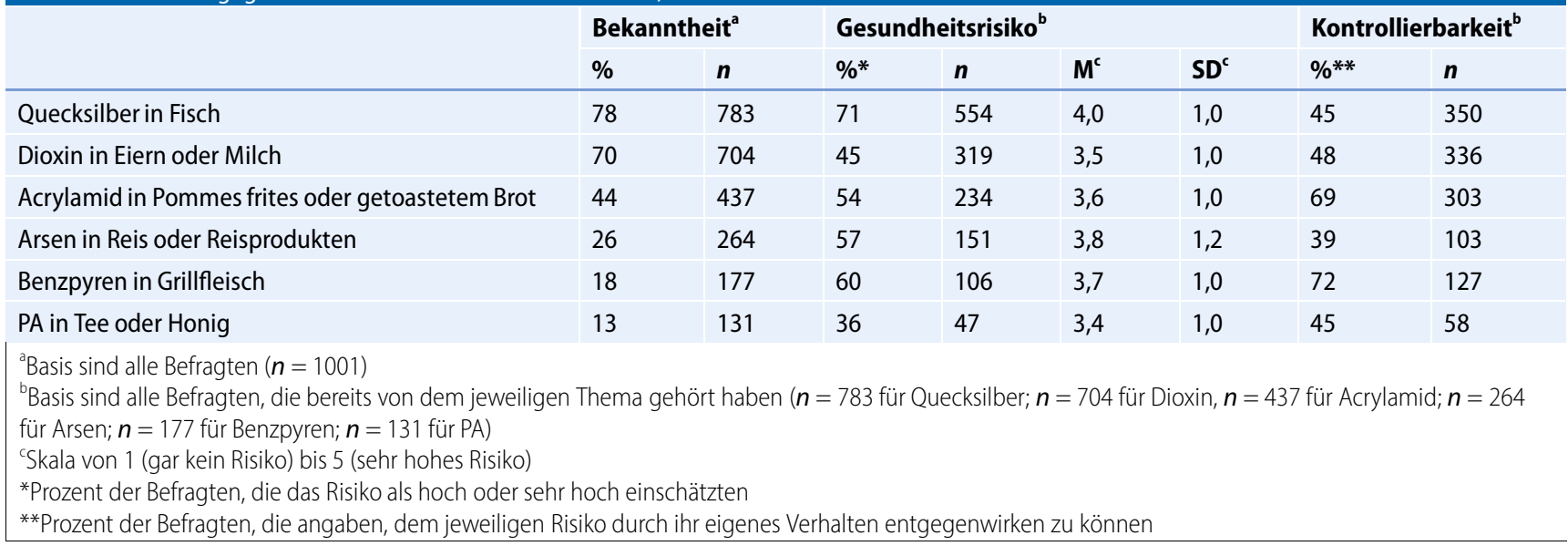

\section{Diskussion}

Die Ergebnisse der vorliegenden Studie zeigen, dass die Bevölkerung beim Thema unerwünschte Stoffe in Lebensmitteln spontan eher selten an Kontaminanten im wissenschaftlich-rechtlichen Sinne $[1,2]$ denkt. In Übereinstimmung mit den Ergebnissen internationaler Studien $[12,18]$ sind Verbraucher eher besorgt über gesundheitliche Risiken im Zusammenhang mit Lebensmittelzusatzstoffen und Fleischprodukten. Zur Erfassung der Einstellungen und Verhaltensweisen im Hinblick auf Kontaminanten ist daher eine deutliche begriffliche Trennung $\mathrm{zu}$ Rückständen und Zusatzstoffen und eine laiengerechte sprachliche Aufbereitung wie in der vorliegenden Befragung zu berücksichtigen.

Mit jeweils 2,3\% der Nennungen waren Metalle/Schwermetalle und Mineralöl die am häufigsten spontan aufgeführten Beispiele für Kontaminanten gemäß der im Rahmen der Befragung verwendeten Begriffsabgrenzung. Angesichts der Häufigkeit, mit der Lebensmittelwarnungen $\mathrm{zu}$ Mykotoxinen (Schimmelpilzgiften) vom Europäischen Schnellwarnsystem RASFF herausgegeben werden - im Jahr 2015 waren es 475 Meldungen [24] - überrascht die geringe ungestützte Bekanntheit dieser Thematik in der Bevölkerung. Lediglich 3 der 1001 Befragten führen spontan Schimmelpilzgifte als Beispiele für unerwünschte Stoffe in Lebensmitteln auf, obwohl das vergleichsweise häufige Auftreten dieser Form der Kontamination eher eine größere Bekanntheit vermuten lässt (beispielsweise durch direkte $\mathrm{Be}$ troffenheit oder aufgrund einer medialen Auseinandersetzung mit der Thematik [25]).

Knapp $60 \%$ der Befragten schätzen Kontaminanten in Lebensmitteln gemäß der verwendeten Begriffserläuterung als ein erhebliches Gesundheitsrisiko ein. Ähnlich wie in Befragungen $\mathrm{zu}$ anderen Themen aus dem Bereich des gesundheitlichen Verbraucherschutzes, insbesondere im Hinblick auf die Qualität und Sicherheit von Lebensmitteln [4], zeigt sich, dass die Bevölkerung die Belastung von Lebensmitteln mit unerwünschten Stoffen als ein tendenziell zunehmendes Risiko wahrnimmt. Dies deckt sich mit den Ergebnissen der von der Europäischen Kommission durchgeführten Eurobarometerbefragung, die eine tendenziell zunehmende Besorgnis über Stoffe wie Quecksilber und Dioxin in Lebensmitteln in den meisten Ländern der Europäischen Union konstatierte [3].

Etwa die Hälfte der Befragten gibt an, sehr bewusst mit belasteten Lebensmitteln umzugehen und Lebensmittel zu meiden, denen eine hohe Belastung mit unerwünschten Stoffen nachgesagt wird. Für die andere Hälfte der Befragten spielt das Thema im Alltag dahingegen keine Rolle oder sind Informationen $\mathrm{zu}$ unerwünschten Stoffen nicht ausschlaggebend für Kaufentscheidungen oder $\mathrm{Zu}$ - bereitungsweisen. Es ist jedoch zu berücksichtigen, dass dieser Aufteilung sozial erwünschte Antwortmuster zugrunde liegen können, sodass die aktive Vermeidung belasteter Lebensmittel im Alltag möglicherweise seltener praktiziert wird als berichtet. Ein ähnliches Antwortmuster konnte auch schon bei zwei Befragungen zu Rückständen von Pflanzenschutzmitteln in Lebensmitteln beobachtet werden $[4,26]$.

Fleisch und Fleischprodukte wurden in der offenen Nachfrage am häufigsten als Beispiele für gemiedene Lebensmittel angeführt, in Übereinstimmung mit den Ergebnissen einer qualitativen Studie in verschiedenen europäischen Ländern und Brasilien, laut derer die Bevölkerung vor allem bei Fleisch und Fleischprodukten ein hohes Gesundheitsrisiko durch Lebensmittelkontaminationen vermutet [12]. Dass die Befragten vermutlich auch Rückstände von Arzneimittel oder Hormonpräparate als Kontaminanten deuten, ist bei der Interpretation dieser Ergebnisse zu bedenken.

Kontaminanten mit einem hohen Bekanntheitsgrad in der Bevölkerung sind Quecksilber in Fisch (78\%) und Dioxin in Eiern oder Milch (70\%). Von Pyrrolizidinalkaloiden (PA) in Honig oder Tee haben dahingegen nur $13 \%$ der Befragten gehört und lediglich $36 \%$ derer, die von PA gehört haben, stufen diesen Stoff als ein bedeutsames gesundheitliches Risiko ein. Am Beispiel von PA wird deutlich, dass eine wissenschaftli- 


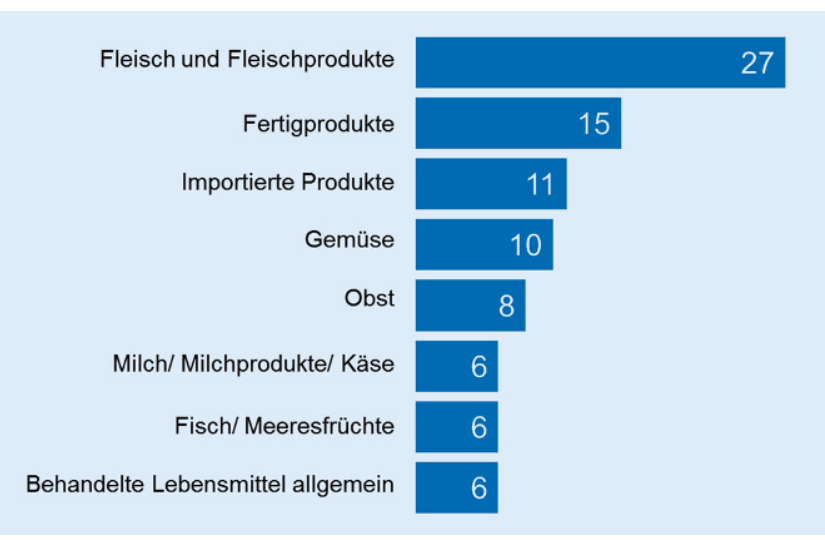

Abb. $1 \Delta$ Anteil der Befragten, die die jeweiligen Lebensmittel wegen ihre (mutmaßlichen) Belastung mit unerwünschten Stoffen meiden (in \% der 495 Befragten, die bewusst Lebensmittel meiden): „Können Sie mir sagen, welche Lebensmittel Sie [im Zusammenhang mit unerwünschten Stoffen] vor allem meiden?"

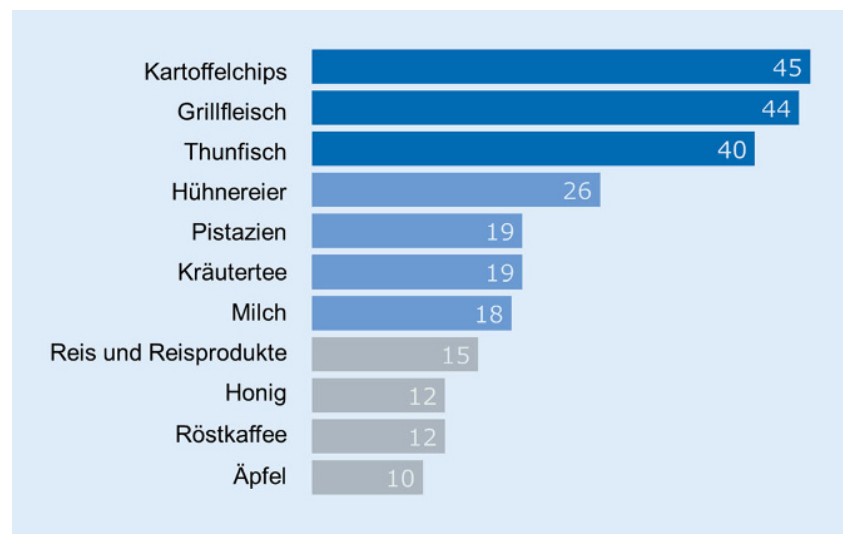

Abb. $2 \Delta$ Anteil der Befragten, der bei 11 Lebensmitteln ein hohes oder sehr hohes Gesundheitsrisiko durch unerwünschte Stoffe vermutet (in \% aller 1001 Befragten): „Inwiefern besteht Ihres Wissens bei den folgenden Lebensmitteln ein Gesundheitsrisiko durch unerwünschte Stoffe?" che Auseinandersetzung mit möglichen Gesundheitsrisiken von Kontaminanten in Lebensmitteln [27-29] nicht zwingend mit deren jeweiliger Bekanntheit in der Bevölkerung einhergeht. Die in der Risikowahrnehmungsforschung bekannte Kombination von weitreichender Unbekanntheit und niedriger Risikoeinschätzung bei der Bevölkerung [7] zeigt, dass insbesondere bei dieser Thematik ein deutlicher Handlungsbedarf besteht.

Aus Sicht der Befragten obliegt die Verantwortung zur Vermeidung gesundheitlicher Risiken durch Kontaminanten in erster Linie der Lebensmittelindustrie und dem Lebensmittelhandel. Im Hinblick auf einige Kontaminanten, wie beispielsweise Acrylamid und Benzpyren, können Verbraucher aber auch durch die Art der Zubereitung der Entstehung dieser gesundheitsschädlichen Stoffe entgegenwirken [14, 30, 31]. Die Befragungsergebnisse zeigen, dass die verbraucherseitige Kontrollierbarkeit der Gesundheitsrisiken von Acrylamid und Benzpyren in der Tat höher eingeschätzt wird als die der übrigen Kontaminanten. Hiermit liefern die Befragungsergebnisse eine sinnvolle Ergänzung zu früheren Studien, die sich hauptsächlich auf die Besorgnis über derartige Stoffe richteten, ungeachtet bedeutsamer Kontextfaktoren wie deren Bekanntheit und wahrgenommene Kontrollierbarkeit [19]. Bei möglichen Kontrollmaßnahmen denkt die Mehrheit der Befragten in erster Linie an einen Verzicht auf betroffene
Lebensmittel oder eine Reduktion des Konsums. Im Hinblick auf Benzpyren und Acrylamid nannte aber mindestens ein Drittel der Befragten Kontrollmaßnahmen, die sich auf die Art der $\mathrm{Zu}$ bereitung bezogen (z. B. „Fett nicht in die Glut tropfen lassen“ oder „Toastbrot nicht zu dunkel werden lassen“).

Die Untersuchung hat insgesamt gezeigt, dass die Bevölkerung Kontaminanten zwar als ein hohes Gesundheitsrisiko einstuft, sie aber selten spontan als Beispiele für unerwünschte Stoffe in Lebensmitteln anführt. Allerdings gibt es Unterschiede zwischen den verschiedenen Kontaminanten hinsichtlich der Bekanntheit in der Bevölkerung. Besonders Pyrrolizidinalkaloide (PA) in Tee oder Honig als vergleichsweise junges Verbraucherschutzthema sind der Bevölkerung weitestgehend unbekannt. Zugleich hat die Studie auch erkennen lassen, dass Personen mit einem niedrigeren Wissensstand zu unerwünschten Stoffen weniger Interesse an Sachinformationen zu diesem Thema bekunden als Personen mit einem höheren Wissensstand ${ }^{2}$.

\footnotetext{
2 Der festgestellte positive Zusammenhang zwischen Informationsstand und Informationsinteresse kann auch (zum Teil) ein Artefakt der Interviewsituation sein. Aus dem Bestreben heraus, in sich schlüssige und konsistente Antworten zu geben, wird das eigene Informationsinteresse möglicherweise aus dem Wissensstand abgeleitet, gemäß der Logik, dass Informiertheit eine generelle Affinität mit der Thematikimpliziert.
}

Vor dem eingangs formulierten Hintergrund der Zielsetzung der Studie, aktuelle Daten zur Wahrnehmung von Kontaminanten in der Lebensmittelkette $\mathrm{zu}$ erheben, um aus diesen mögliche Anknüpfungspunkte und Handlungsbedarf für eine zielgruppengerechte Risikokommunikation abzuleiten, erwächst aus diesen Ergebnissen eine Herausforderung in doppelter Hinsicht. Wie bereits in anderen Studien hat sich auch hier eine Definitionsbarriere zwischen Laien und Experten gezeigt, da es in der Bevölkerung keine homogene Wahrnehmung von Kontaminanten gibt, sondern diese zum Teil höchst unterschiedlich wahrgenommen werden sowohl im Hinblick auf ihr Risikopotenzial wie auch hinsichtlich ihrer Kontrollierbarkeit durch die Verbraucher. Hier gilt es künftig, ein kohärentes Verständnis von Lebensmittelkontaminanten in der Laienöffentlichkeit zu schaffen, bei gleichzeitiger Berücksichtigung und Vermittlung des Umstands, dass sich diese hinsichtlich möglicher individueller Maßnahmen zur Risikominimierung unterscheiden. Des Weiteren müssen vor allem weniger gut informierte Personengruppen zunächst im Sinne eines „awareness-raising“ erreicht werden. Einen möglichen Anknüpfungspunkt bieten dabei beispielsweise Multiplikatoren im Gesundheitssektor, die in ihrem Berufsalltag persönlichen Umgang mit verschiedensten Bevölkerungsgruppen pflegen und somit Informationen einer breiten Öffentlichkeit zugäng- 
lich machen können. Generell kann eine verstärkte Zusammenarbeit mit Multiplikatoren aus verschiedenen gesellschaftlichen Feldern dazu beitragen, die Reichweite risikorelevanter Informationen $\mathrm{zu}$ erhöhen.

\section{Korrespondenzadresse}

\section{Dr. S. Koch}

Bundesinstitut für Risikobewertung

Max-Dohrn-Straße 8-10, 10589 Berlin,

Deutschland

Severine.Koch@bfr.bund.de

Danksagung. Wir danken Judith Probstmeyer und Jürgen Thier-Kundke für ihre Unterstützung bei der Erstellung dieses Beitrags.

\section{Einhaltung ethischer Richtlinien}

Interessenkonflikt. S. Koch, M. Lohmann, A. Epp und G.-F. Böl geben an, dass kein Interessenkonflikt besteht.

Es wurden keine Untersuchungen an Patienten oder Tieren durchgeführt. Die Befragungsdaten wurden anonym und unter Einhaltung ethischer Richtlinien erhoben.

Open Access. Dieser Artikel wird unter der Creative Commons Namensnennung 4.0 International Lizenz (http://creativecommons.org/licenses/by/4.0/deed. de) veröffentlicht, welche die Nutzung, Vervielfältigung, Bearbeitung, Verbreitung und Wiedergabe in jeglichem Medium und Format erlaubt, sofern Sie den/die ursprünglichen Autor(en) und die Quelle ordnungsgemäß nennen, einen Link zur Creative Commons Lizenz beifügen und angeben, ob Änderungen vorgenommen wurden.

\section{Literatur}

1. Council Regulation (EEC) (1993) No 315/93 laying down community procedures for contaminants in food, article 1. http://eur-lex.europa.eu/eli/reg/ 1993/315/2009-08-07.Zugegriffen: 19. Dez 2016

2. European Commission (2008) Factsheet food contaminants. Health and consumer protection directorate-general. https://ec.europa.eu/food/ sites/food/files/safety/docs/cs_contaminants_ factsheet_en.pdf.Zugegriffen:12.Dez 2016

3. Eurobarometer (2010) Food-related risks. Special Eurobarometer 354/wave 73.5-TNS opinion and social. The European Commission. http:// ec.europa.eu/public_opinion/archives/ebs/ebs 354_en.pdf.Zugegriffen: 13.Dez 2016

4. Bundesinstitut für Risikobewertung (2016) BfRVerbrauchermonitor 2016 - Spezial Pflanzenschutzmittel. http://www.bfr.bund.de/cm/350/ bfr-verbrauchermonitor-2016.pdf. Zugegriffen: 12. Dez 2016

5. Renn O (2014) Das Risikoparadox - Warum wir uns vor dem Falschen fürchten. Fischer, Frankfurt am Main

6. Rossmann C, Brosius HB (2013) The perils of risk communication and the role of the mass media.
Bundesgesundheitsblatt Gesundheitsforschung Gesundheitsschutz 56:118-123

7. Slovic P (1987) Perception of risk. Science 236:280-285

8. Slovic $P$ (2000) The perception of risk. Earthscan, London

9. Wilkinson SBT, Rowe G, Lambert N (2004) The risks of eating and drinking. EMBO Rep 5:S27-S31

10. Kurzenhäuser S, Epp A (2009) Wahrnehmung von gesundheitlichen Risiken - Psychologische und soziale Einflussfaktoren. Bundesgesundheits blatt Gesundheitsforschung Gesundheitsschutz 52:1141

11. Schütz H, Wiedemann PM (2003) Risikowahrnehmung in der Gesellschaft. Bundesgesundheitsblatt Gesundheitsforschung Gesundheitsschutz 46:549-554

12. Kher SV, De Jonge J, Wentholt MTA et al (2013) Consumer perceptions of risks of chemical and microbiological contaminants associated with food chains: a cross-national study. Int J Consum Stud 37:73-83

13. Kennedy J, Delaney L, Hudson EM, Mcgloin A, Wall PG (2010) Public perceptions of the dioxin incident in Irish pork. J Risk Res 13:937-949

14. Epp A (2007) Acrylamid in Lebensmitteln ein Risiko im Bereitschaftsmodus. Umweltmed Informationsd 1/2007:5-9

15. Dickson-Spillmann M, Siegrist M, Keller C, Wormuth M (2009) Phthalate exposure through food and consumers' risk perception of chemicals in food. Risk Analysis 29:1170-1181

16. Dickson-Spillmann M, Siegrist M, Keller C (2011) Attitudes toward chemicals are associated with preference for natural food. Food Qual Prefer 22:149-156

17. Degen GH (2003) Einige Anmerkungen zur Risikokommunikation über Chemikalien. Umweltmed Forsch Prax 8:330-334

18. Buchler S, Smith K, Lawrence G (2010) Food risks, old and new - demographic characteristics and perceptions of food additives, regulation and contamination in Australia. J Sociol 46:353-374

19. Eurobarometer (2006) Risk issues. Special Eurobarometer 238/wave 64.1-TNS opinion and social. The European Commission. http://ec.europa.eu/ public_opinion/archives/ebs/ebs_238_en.pdf. Zugegriffen: 13.Dez 2016

20. Borra ST, Bouchoux A (2009) Effects of science and the media on consumer perceptions about dietary sugars. J Nutr 139:1214S-1218S

21. Diekmann A (2007) Empirische Sozialforschung Grundlagen, Methoden, Anwendungen. Rowohlt, Reinbek bei Hamburg

22. Gaziano C (2005) Comparative analysis of withinhousehold respondent selection techniques. Public Opin Q 69:124-157

23. Gabler S, Ayhan Ö (2007) Gewichtung bei Erhebungen in Festnetz und über Mobilfunk: Ein Dual Frame Ansatz. In: Gabler S, Häder S (Hrsg) Mobilfunktelefonie - Eine Herausforderung für die Umfrageforschung. GESIS-ZUMA, Mannheim, S39-45

24. European Commission (2016) RASFF - The Rapid Alert System for Food and Feed - 2015 annual report. https://ec.europa.eu/food/sites/food/ files/safety/docs/rasff_annual_report_2015.pdf. Zugegriffen: 13.Dez 2016

25. Zeit Online (2016) Stiftung Warentest - Schimmelpilzgifte in Nuss-Nugat-Cremes. http://www. zeit.de/wissen/gesundheit/2016-03/stiftungwarentest-schimmelpilz-gift-nuss-nougatcreme. Zugegriffen:22. Febr 2017
26. Epp A, Michalski B, Banasiak U, Böl GF (2010) Pflanzenschutzmittel-Rückstände in Lebensmitteln. Die Wahrnehmung der deutschen Bevölkerung - Ein Ergebnisbericht. BfR-Wissenschaft 07/2010, Berlin. http://www.bfr.bund.de/ cm/350/pflanzenschutzmittel_rueckstaende_in_ lebensmitteln.pdf.Zugegriffen:31. Jan 2017

27. Bundesinstitut für Risikobewertung (2011) Analytik und Toxizität von Pyrrolizidinalkaloiden sowie eine Einschätzung des gesundheitlichen Risikos durch deren Vorkommen in Honig. http://www.bfr. bund.de/cm/343/analytik-und-toxizitaet-vonpyrrolizidinalkaloiden.pdf. Zugegriffen: 19. Dez 2016

28. Bundesinstitut für Risikobewertung (2016) Pyrrolizidinalkaloide: Gehalte in Lebensmitteln sollen nach wie vor so weit wie möglich gesenkt werden. http://www.bfr.bund.de/cm/343/ pyrrolizidinalkaloide-gehalte-in-lebensmittelnsollen-nach-wie-vor-so-weit-wie-moeglichgesenkt-werden.pdf.Zugegriffen: 19. Dez 2016

29. Bundesinstitut für Risikobewertung (2007) Salatmischung mit Pyrrolizidinalkaloid-haltigem Greiskraut verunreinigt. http://www.bfr.bund.de/cm/ 343/salatmischung_mit_pyrrolizidinalkaloid_ haltigem_geiskraut_verunreinigt.pdf. Zugegriffen: 19. Dez 2016

30. Bundesinstitut für Risikobewertung (2016) Ausgewählte Fragen und Antworten zum Grillen. http://www.bfr.bund.de/cm/343/ausgewaehltefragen-und-antworten-zum-grillen.pdf. Zugegriffen: 02. Febr 2017

31. Bundesinstitut für Risikobewertung (2011) Acrylamid in Lebensmitteln. http://www.bfr. bund.de/cm/343/acrylamid-in-lebensmitteln. pdf. Zugegriffen:02. Febr 2017 